\title{
Éditorial
}

\section{Chromothripsie, et pourquoi pas chromosomothripsie ?}

\author{
Jacky Samson
}

Rédacteur en chef

\author{
«Le néologisme, c'est la langue qui fait ses besoins » Frédéric Dard
}

La carcinogenèse n'avait presque plus de secrets. Avec la confirmation de la théorie clonale - hypothèse émise dans les années 50 et brillamment exposée par Nowell en 1976 dans un article [10] qui fait toujours référence - et la découverte des oncogènes et des gènes suppresseurs de tumeur, on pensait être proche du but et avoir presque tout compris. Le cancer était considéré comme une maladie multigénique, résultant de l'altération de 10 à 20 gènes. Ces altérations se font par étapes successives et 4 à 7 mutations ou événements stochastiques seraient nécessaires pour arriver à la cellule cancéreuse. Chaque étape donne naissance à des cellules ayant acquis un avantage sélectif (terme emprunté à la théorie de l'évolution) et l'une d'entre elles passe à l'étape suivante. La transformation maligne est donc un processus dynamique qui, à chaque étape, sélectionne une cellule ayant subi une ou plusieurs altérations. Ce processus aboutit à la formation d'une cellule cancéreuse, c'est-à-dire d'une cellule qui a acquis au moins 6 propriétés qui toutes favorisent la croissance tumorale [4] : indépendance vis-à-vis des signaux stimulant la croissance, insensibilité aux signaux inhibant la croissance, disparition du processus de mort cellulaire programmée (apoptose), potentiel illimité de prolifération, stimulation de l'angiogenèse et pouvoir d'invasion des tissus et de donner des métastases. La carcinogenèse serait donc un processus gradualiste et quelques auteurs n'ont pas hésité à établir un paralléle avec la théorie de l'évolution de Darwin [4, 12], certains allant même jusqu'à comparer la carcinogenèse à une spéciation [2].

Devant ces progrès, nous étions arrivés à un tel état de quiétude intellectuelle que nous avions presque oublié que les cancers ne résultent probablement pas toujours d'une succession de mutations intervenant sur une période allant de 5 à 20 ans. En janvier 2011, brutal retour à la réalité. Dans un éditorial bien documenté [9] pour préparer le lecteur à une découverte qui bouscule la conception quasi dogmatique de la carcinogenèse, on nous annonce un cataclysme en carcinologie : le cancer pourrait dans certains cas résulter de la pulvérisation cataclysmique d'un chromosome [11]. Dans ce nouveau processus de carcinogenèse, on observe des dizaines ou des centaines de réarrangements intéressant uniquement une région chromosomique étroite, et non pas l'ensemble du génome, comme s'il y avait eu une fragmentation de la région suivie d'une reconstitution aléatoire donc aberrante. La grande majorité de ces réarrangements semble se produire lors d'un événement unique car le nombre de copies des fragments n'excède pas 2 ou 3 dans le chromosome remanié. Ce processus a été retrouvé dans 2 à $3 \%$ des cancers et dans $25 \%$ des tumeurs osseuses étudiés. De nombreuses questions persistent, en particulier la cause et la chronologie de ce cataclysme restent inconnues. Les auteurs rapprochent ce phénomène des changements rapides décrits dans les équilibres ponctués, théorie proposée en 1972 par deux paléontologues américains, Niles Eldredge et Stephen Jay Gould, pour expliquer l'absence ou la rareté, dans certains cas, des formes intermédiaires liant une espèce à l'autre dans les archives fossilifères [3]. Cette hypothèse a suscité de vives controverses et Gould a répondu, entre autres aux darwiniens, par cette métaphore : «Il s'agit d'ajouter de nombreuses extensions à l'édifice de la théorie de l'évolution darwinienne, quitte, certes, à en modifier profondément l'aspect extérieur, mais en en conservant soigneusement les fondations ». [6]. Peu après, un autre article [8] a confirmé l'existence du phénomène de pulvérisation cataclysmique dans le myélome multiple et montré qu'il était associé à un pronostic péjoratif. Ce phénomène a été également retrouvé dans certaines maladies congénitales où il pourrait survenir lors de la réplication de l'ADN au cours de la gamétognèse ou à un stade postzygotique $[5,7]$. Les réarrangements génomiques complexes résultant de ce phénomène sont donc impliqués dans la formation et la progression des cancers et dans l'étiologie de maladies congénitales. 
Pour nommer ce phénomène, les auteurs ont eu recours à un néologisme : « chromothripsis (Greek, chromos for chromosome ; thripsis, shaterring into pieces) » [11]. Ce terme, et sa version française chromothripsie, a été repris dans les différentes publications sans que quiconque - comme les 32 auteurs de l'article initial et les reviewers de cet article - ne reléve que chromos en grec signifie couleur et non chromosome ! Après un petit rappel historique, l'erreur devient plus saisissante. Initialement, les chromosomes ont été surtout étudiés par Walther Flemmning, Eduard Strasburger et Teodor Boveri (qui sera le $1^{\mathrm{er}}$ en 1914 à formuler l'hypotèse de l'origine génétique des cancers ). Différents termes étaient alors utilisés : éléments chromatiques, boucles nucléaires, karyosomes, segments nucléaires ou idants. Le terme chromosome (du grec khrôma pour couleur et sôma pour corps) fut proposé 1888 par Wilhelm Waldeyer pour désigner ces filaments basophiles colorés [1, 13]. Les auteurs de l'article princeps auraient donc dû logiquement proposer le terme de chromosomothripsis mais ce terme est moins élégant, trop long, plus difficile à prononcer et sonne beaucoup moins bien que chromothripsie. On aurait pu croire qu'ils avaient tout simplement réalisé une pulvérisation cataclysmique du mot chromosome, s'ils n'avaient pas écrit en toutes lettres « chromos for chromosome »?

Soyons reconnaissants pour cette découverte et oublions cette faute vénielle. Remercions les auteurs d'avoir proposé un terme attrayant dont la magie a déjà opéré puisque l'erreur est jusque-là passée inaperçue. Et puis, il y a déjà en Médecine bien d'autres imprécisions avec les néologismes ayant une racine grecque ou latine: l'exemple sans doute le plus connu reste apoptose (chute des feuilles en grec) pour mort cellulaire programmée. Chromothripsie aura probablement la même diffusion et la même reconnaissance qu'apoptose, en partie grâce à un barbarisme.

\section{Conflits d'intérêt : aucun}

\section{Références}

1. Cremer T, Cremer C. Centennial of Wilhem Waldeyer's introduction of the term "chromosome" in 1888. Cytogenet Cell Genet 1988;48:66-7.

2. Duesberg P, Mandrioli D, McCormack A, Nicholson JM. Is carcinogenisis a form of speciation ? Cell Cycle 2011;10:2100-4.

3. Eldredge N, Gould SJ. Punctuated equilibria: an alternative to phyletic gradualism (pp. 82-115). In: Schopf TJM ed. Models in paleobiology. Freeman Cooper, San Francisco, 1972.

4. Hanaghan D, Weinberg RA. The hallmarks of cancer. Cell 2000;100:57-70.

5. Kloosterman WP, Guryev V, Van Roosmalen M, Duran KJ, De Bruijn E, Bakker SCM, Letteboer T, Van Nesselrooij B, Hochstenbach R, Poot M, Cuppen E. Chromotripsis as a mechanism driving complex de novo structural rearrangements in the germline. Hum Mol Gen 2011;20:1916-24.

6. Langlois C. La structure de la théorie de l'évolution, de Stephen Jay Gould. http://planet-terre.ens-lyon.fr

7. Liu P, et al. (19 co-auteurs). Chromosome catastrophes involve replication mechanisms generating complex genomic rearrangements. Cell 2011;146:889-903.

8. Magrangeas F, Avet-Loiseau H, Munshi NC, Minvielle S. Chromothripsis identifies a rare and aggressive entity among newly diagnosed multiple myeloma patients. Blood 2011;118:675-8.

9. Meyerson M, Pellman D. Cancer genomes evolve by pulverizing single chromosomes. Cell 2011;144:9-10.

10. Novell PC. The clonal evolution of tumor cell populations. Science 1976;194:23-8.

11. Stephens PJ, et al. (31 co-auteurs). Massive genomic rearrangement acquired in a single catastrophic event during cancer development. Cell 2011;144:27-40.

12. Vineis $P$, Berwick $M$. The population dynamics of cancer: a Darwinian perspective. Int J Epidemiol 2006;35:1151-9.

13. Winkelmann A. Wilhelm von Waldeyer-Hartz (1836-1921): an anatomist who left his mark. Clin Anat 2007;20:231-4. 